CERN-EP/2000-131

17 October 2000

\title{
Searches for neutral Higgs bosons in $\mathrm{e}^{+} \mathrm{e}^{-}$collisions at centre-of-mass energies from 192 to $202 \mathrm{GeV}$
}

\author{
The ALEPH Collaboration*)
}

\begin{abstract}
Searches for neutral Higgs bosons are performed with the $237 \mathrm{pb}^{-1}$ of data collected in 1999 by the ALEPH detector at LEP, for centre-of-mass energies between 191.6 and 201.6 GeV. These searches apply to Higgs bosons within the context of the Standard Model and its minimal supersymmetric extension (MSSM) as well as to invisibly decaying Higgs bosons. No evidence of a signal is seen. A lower limit on the mass of the Standard Model Higgs boson of $107.7 \mathrm{GeV} / c^{2}$ at $95 \%$ confidence level is set. In the MSSM, lower limits of 91.2 and $91.6 \mathrm{GeV} / c^{2}$ are derived for the masses of the neutral Higgs bosons $\mathrm{h}$ and A, respectively. For a Higgs boson decaying invisibly and produced with the Standard Model cross section, masses below $106.4 \mathrm{GeV} / c^{2}$ are excluded.
\end{abstract}

(To be submitted to Physics Letters)

*) See next pages for the list of authors. 


\section{The ALEPH Collaboration}

R. Barate, D. Decamp, P. Ghez, C. Goy, S. Jezequel, J.-P. Lees, F. Martin, E. Merle, M.-N. Minard, B. Pietrzyk

Laboratoire de Physique des Particules (LAPP), IN $N^{2} P^{3}$-CNRS, F-74019 Annecy-le-Vieux Cedex, France

S. Bravo, M.P. Casado, M. Chmeissani, J.M. Crespo, E. Fernandez, M. Fernandez-Bosman, Ll. Garrido, ${ }^{15}$

E. Graugés, J. Lopez, M. Martinez, G. Merino, R. Miquel, Ll.M. Mir, A. Pacheco, D. Paneque, H. Ruiz Institut de Física d'Altes Energies, Universitat Autònoma de Barcelona, E-08193 Bellaterra (Barcelona), Spain ${ }^{7}$

A. Colaleo, D. Creanza, N. De Filippis, M. de Palma, G. Iaselli, G. Maggi, M. Maggi, ${ }^{1}$ S. Nuzzo, A. Ranieri, G. Raso ${ }^{24}$ F. Ruggieri, G. Selvaggi, L. Silvestris, P. Tempesta, A. Tricomi, ${ }^{3}$ G. Zito

Dipartimento di Fisica, INFN Sezione di Bari, I-70126 Bari, Italy

X. Huang, J. Lin, Q. Ouyang, T. Wang, Y. Xie, R. Xu, S. Xue, J. Zhang, L. Zhang, W. Zhao Institute of High Energy Physics, Academia Sinica, Beijing, The People's Republic of China ${ }^{8}$

D. Abbaneo, P. Azzurri, G. Boix, ${ }^{6}$ O. Buchmüller, M. Cattaneo, F. Cerutti, B. Clerbaux, G. Dissertori, H. Drevermann, R.W. Forty, M. Frank, F. Gianotti, T.C. Greening, J.B. Hansen, J. Harvey, D.E. Hutchcroft, P. Janot, B. Jost, M. Kado, V. Lemaitre, P. Maley, P. Mato, A. Minten, A. Moutoussi, F. Ranjard, L. Rolandi, D. Schlatter, M. Schmitt, ${ }^{20}$ O. Schneider, ${ }^{2}$ P. Spagnolo, W. Tejessy, F. Teubert, E. Tournefier, ${ }^{26}$ A. Valassi, J.J. Ward, A.E. Wright

European Laboratory for Particle Physics (CERN), CH-1211 Geneva 23, Switzerland

Z. Ajaltouni, F. Badaud, S. Dessagne, A. Falvard, D. Fayolle, P. Gay, P. Henrard, J. Jousset, B. Michel, S. Monteil, J-C. Montret, D. Pallin, J.M. Pascolo, P. Perret, F. Podlyski

Laboratoire de Physique Corpusculaire, Université Blaise Pascal, IN ${ }^{2} P^{3}-C N R S$, Clermont-Ferrand, F-63177 Aubière, France

J.D. Hansen, J.R. Hansen, P.H. Hansen, B.S. Nilsson, A. Wäänänen

Niels Bohr Institute, 2100 Copenhagen, DK-Denmark ${ }^{9}$

G. Daskalakis, A. Kyriakis, C. Markou, E. Simopoulou, A. Vayaki

Nuclear Research Center Demokritos (NRCD), GR-15310 Attiki, Greece

A. Blondel, ${ }^{12}$ J.-C. Brient, F. Machefert, A. Rougé, M. Swynghedauw, R. Tanaka H. Videau

Laboratoire de Physique Nucléaire et des Hautes Energies, Ecole Polytechnique, IN $\mathrm{IN}^{2} \mathrm{P}^{3}$ CNRS, F-91128 Palaiseau Cedex, France

E. Focardi, G. Parrini, K. Zachariadou

Dipartimento di Fisica, Università di Firenze, INFN Sezione di Firenze, I-50125 Firenze, Italy

A. Antonelli, M. Antonelli, G. Bencivenni, G. Bologna, ${ }^{4}$ F. Bossi, P. Campana, G. Capon, V. Chiarella, P. Laurelli, G. Mannocchi, ${ }^{5}$ F. Murtas, G.P. Murtas, L. Passalacqua, M. Pepe-Altarelli ${ }^{25}$

Laboratori Nazionali dell'INFN (LNF-INFN), I-00044 Frascati, Italy

M. Chalmers, A.W. Halley, J. Kennedy, J.G. Lynch, P. Negus, V. O'Shea, B. Raeven, D. Smith, P. Teixeira-Dias, A.S. Thompson

Department of Physics and Astronomy, University of Glasgow, Glasgow G12 8QQ,United Kingdom ${ }^{10}$

R. Cavanaugh, S. Dhamotharan, C. Geweniger, P. Hanke, V. Hepp, E.E. Kluge, G. Leibenguth, A. Putzer, K. Tittel, S. Werner, ${ }^{19}$ M. Wunsch ${ }^{19}$

Kirchhoff-Institut für Physik, Universität Heidelberg, D-69120 Heidelberg, Germany ${ }^{16}$ 
R. Beuselinck, D.M. Binnie, W. Cameron, G. Davies, P.J. Dornan, M. Girone, ${ }^{1}$ N. Marinelli, J. Nowell, H. Przysiezniak, J.K. Sedgbeer, J.C. Thompson, ${ }^{14}$ E. Thomson, ${ }^{23}$ R. White

Department of Physics, Imperial College, London SW7 2BZ, United Kingdom ${ }^{10}$

V.M. Ghete, P. Girtler, E. Kneringer, D. Kuhn, G. Rudolph

Institut für Experimentalphysik, Universität Innsbruck, A-6020 Innsbruck, Austria ${ }^{18}$

E. Bouhova-Tracker, C.K. Bowdery, P.G. Buck, D.P. Clarke, G. Ellis, A.J. Finch, F. Foster, G. Hughes, R.W.L. Jones, ${ }^{1}$ N.A. Robertson, M. Smizanska

Department of Physics, University of Lancaster, Lancaster LA1 4YB, United Kingdom ${ }^{10}$

I. Giehl, F. Hölldorfer, K. Jakobs, K. Kleinknecht, M. Kröcker, A.-S. Müller, H.-A. Nürnberger, G. Quast, ${ }^{1}$

B. Renk, E. Rohne, H.-G. Sander, S. Schmeling, H. Wachsmuth, C. Zeitnitz, T. Ziegler

Institut für Physik, Universität Mainz, D-55099 Mainz, Germany ${ }^{16}$

A. Bonissent, J. Carr, P. Coyle, C. Curtil, A. Ealet, D. Fouchez, O. Leroy, T. Kachelhoffer, P. Payre, D. Rousseau, A. Tilquin

Centre de Physique des Particules de Marseille, Univ Méditerranée, IN² P3_CNRS, F-13288 Marseille, France

M. Aleppo, S. Gilardoni, F. Ragusa

Dipartimento di Fisica, Università di Milano e INFN Sezione di Milano, I-20133 Milano, Italy.

A. David, H. Dietl, G. Ganis, ${ }^{27}$ K. Hüttmann, G. Lütjens, C. Mannert, W. Männer, H.-G. Moser, S. Schael, R. Settles, ${ }^{1}$ H. Stenzel, W. Wiedenmann, G. Wolf

Max-Planck-Institut für Physik, Werner-Heisenberg-Institut, D-80805 München, Germany ${ }^{16}$

J. Boucrot, ${ }^{1}$ O. Callot, M. Davier, L. Duflot, J.-F. Grivaz, Ph. Heusse, A. Jacholkowska, ${ }^{1}$ L. Serin, J.-J. Veillet,

I. Videau, J.-B. de Vivie de Régie, C. Yuan, D. Zerwas

Laboratoire de l'Accélérateur Linéaire, Université de Paris-Sud, IN $N^{2} P^{3}$-CNRS, F-91898 Orsay Cedex, France

G. Bagliesi, T. Boccali, G. Calderini, V. Ciulli, L. Foà, A. Giammanco, A. Giassi, F. Ligabue, A. Messineo, F. Palla, ${ }^{1}$ G. Sanguinetti, A. Sciabà, G. Sguazzoni, R. Tenchini, ${ }^{1}$ A. Venturi, P.G. Verdini

Dipartimento di Fisica dell'Università, INFN Sezione di Pisa, e Scuola Normale Superiore, I-56010

Pisa, Italy

G.A. Blair, J. Coles, G. Cowan, M.G. Green, L.T. Jones, T. Medcalf, J.A. Strong,

J.H. von Wimmersperg-Toeller

Department of Physics, Royal Holloway \& Bedford New College, University of London, Surrey TW20 OEX, United Kingdom ${ }^{10}$

R.W. Clifft, T.R. Edgecock, P.R. Norton, I.R. Tomalin

Particle Physics Dept., Rutherford Appleton Laboratory, Chilton, Didcot, Oxon OX11 OQX, United Kingdom $^{10}$

B. Bloch-Devaux, ${ }^{1}$ D. Boumediene, P. Colas, B. Fabbro, E. Lançon, M.-C. Lemaire, E. Locci, P. Perez,

J. Rander, J.-F. Renardy, A. Rosowsky, P. Seager, ${ }^{13}$ A. Trabelsi, ${ }^{21}$ B. Tuchming, B. Vallage

CEA, DAPNIA/Service de Physique des Particules, CE-Saclay, F-91191 Gif-sur-Yvette Cedex, France $^{17}$

N. Konstantinidis, C. Loomis, A.M. Litke, G. Taylor

Institute for Particle Physics, University of California at Santa Cruz, Santa Cruz, CA 95064, USA 22

C.N. Booth, S. Cartwright, F. Combley, P.N. Hodgson, M. Lehto, L.F. Thompson

Department of Physics, University of Sheffield, Sheffield S3 7RH, United Kingdom ${ }^{10}$

K. Affholderbach, A. Böhrer, S. Brandt, C. Grupen, J. Hess, A. Misiejuk, G. Prange, U. Sieler

Fachbereich Physik, Universität Siegen, D-57068 Siegen, Germany ${ }^{16}$

C. Borean, G. Giannini, B. Gobbo

Dipartimento di Fisica, Università di Trieste e INFN Sezione di Trieste, I-34127 Trieste, Italy 
H. He, J. Putz, J. Rothberg, S. Wasserbaech

Experimental Elementary Particle Physics, University of Washington, Seattle, WA 98195 U.S.A.

S.R. Armstrong, K. Cranmer, P. Elmer, D.P.S. Ferguson, Y. Gao, S. González, O.J. Hayes, H. Hu, S. Jin, J. Kile, P.A. McNamara III, J. Nielsen, W. Orejudos, Y.B. Pan, Y. Saadi, I.J. Scott, J. Walsh, J. Wu, Sau Lan Wu, X. Wu, G. Zobernig

Department of Physics, University of Wisconsin, Madison, WI 53706, USA ${ }^{11}$

\footnotetext{
${ }^{1}$ Also at CERN, 1211 Geneva 23, Switzerland.

${ }^{2}$ Now at Université de Lausanne, 1015 Lausanne, Switzerland.

${ }^{3}$ Also at Dipartimento di Fisica di Catania and INFN Sezione di Catania, 95129 Catania, Italy.

${ }^{4}$ Deceased.

${ }^{5}$ Also Istituto di Cosmo-Geofisica del C.N.R., Torino, Italy.

${ }^{6}$ Supported by the Commission of the European Communities, contract ERBFMBICT982894.

${ }^{7}$ Supported by CICYT, Spain.

${ }^{8}$ Supported by the National Science Foundation of China.

${ }^{9}$ Supported by the Danish Natural Science Research Council.

${ }^{10}$ Supported by the UK Particle Physics and Astronomy Research Council.

${ }^{11}$ Supported by the US Department of Energy, grant DE-FG0295-ER40896.

${ }^{12}$ Now at Departement de Physique Corpusculaire, Université de Genève, 1211 Genève 4, Switzerland.

${ }^{13}$ Supported by the Commission of the European Communities, contract ERBFMBICT982874.

${ }^{14}$ Also at Rutherford Appleton Laboratory, Chilton, Didcot, UK.

${ }^{15}$ Permanent address: Universitat de Barcelona, 08208 Barcelona, Spain.

${ }^{16}$ Supported by the Bundesministerium für Bildung, Wissenschaft, Forschung und Technologie, Germany.

${ }^{17}$ Supported by the Direction des Sciences de la Matière, C.E.A.

${ }^{18}$ Supported by the Austrian Ministry for Science and Transport.

${ }^{19}$ Now at SAP AG, 69185 Walldorf, Germany

${ }^{20}$ Now at Harvard University, Cambridge, MA 02138, U.S.A.

${ }^{21}$ Now at Département de Physique, Faculté des Sciences de Tunis, 1060 Le Belvédère, Tunisia.

${ }^{22}$ Supported by the US Department of Energy, grant DE-FG03-92ER40689.

${ }^{23}$ Now at Department of Physics, Ohio State University, Columbus, OH 43210-1106, U.S.A.

${ }^{24}$ Also at Dipartimento di Fisica e Tecnologie Relative, Università di Palermo, Palermo, Italy.

25 Now at CFRN 1211 Geneva 23 Switzerland
} 


\section{Introduction}

Searches for neutral Higgs bosons of the Standard Model, its minimal supersymmetric extension (MSSM), and extensions allowing Higgs boson decays into invisible final states were performed using data collected by the ALEPH detector at LEP during 1999. The data sample was taken at four centre-of-mass energies, 191.6, 195.5, 199.5, and $201.6 \mathrm{GeV}$ at which 28.9, 79.9, 86.3, and $41.9 \mathrm{pb}^{-1}$ of data were collected, respectively. The total data sample corresponding to an integrated luminosity of $237 \mathrm{pb}^{-1}$ was analysed to search for topologies arising from the $\mathrm{e}^{+} \mathrm{e}^{-} \rightarrow$ hZ Higgsstrahlung process supplemented by $\mathrm{W}$ and $\mathrm{Z}$ gauge boson fusion, and from the $\mathrm{e}^{+} \mathrm{e}^{-} \rightarrow \mathrm{hA}$ associated pair-production process of the MSSM. The production cross section of the Higgsstrahlung process in the MSSM is reduced by a factor $\sin ^{2}(\beta-\alpha)$, where $\tan \beta$ is the ratio of the vacuum expectation values of the two Higgs doublets and $\alpha$ is the mixing angle in the CP-even Higgs sector. The hA production cross section is proportional to $\cos ^{2}(\beta-\alpha)$. For an invisibly decaying Higgs boson, the observable rate of the Higgsstrahlung process can be expressed as $\xi^{2} \sigma_{\mathrm{SM}}\left(\mathrm{e}^{+} \mathrm{e}^{-} \rightarrow \mathrm{hZ}\right)$, where $\xi^{2}$ is the product of the branching ratio to invisible decays and a model-dependent factor which reduces the cross section with respect to that in the Standard Model.

Searches for neutral Higgs bosons with the ALEPH detector have already been carried out up to a centre-of-mass energy of $188.6 \mathrm{GeV}$ [1, 2]; no evidence of a signal was found. A lower limit at $95 \%$ confidence level (CL) was set at $92.9 \mathrm{GeV} / c^{2}$ on the Standard Model Higgs boson mass. In the MSSM for the benchmark parameter set with maximal stop mixing [3], lower limits of $82.5 \mathrm{GeV} / c^{2}$ and $82.6 \mathrm{GeV} / c^{2}$ were derived on the masses of the $\mathrm{h}$ and A Higgs bosons, respectively. For an invisibly decaying Higgs boson, a lower limit of $95.4 \mathrm{GeV} / c^{2}$ was set, for a production cross section equal to that in the Standard Model. Similar searches have been performed by the other LEP experiments [ [

The higher centre-of-mass energies and integrated luminosity in the 1999 data substantially increase the experimental sensitivity for the detection of Higgs bosons with respect to previous results. Nevertheless, the background processes are the same as those described in Refs. [1, 2]. The theoretical framework, the event selections, the study of systematic uncertainties, and the result extraction are therefore very similar to those previously described. The differences mainly consist of reoptimization of event selections, the introduction of new discriminating variables, and improvements related to the simulation of signal processes.

\section{ALEPH detector}

The components of the ALEPH detector that are most relevant for the analyses presented here are summarized in this section. A more detailed description of the detector can be found in Ref. [5] and its performance in Ref. [6].

Three coaxial tracking devices are located inside a solenoidal superconducting coil which produces an axial magnetic field of $1.5 \mathrm{~T}$. The vertex detector (VDET) [7] consists of two cylindrical layers of silicon wafers situated at average radii of 6.3 and $11.0 \mathrm{~cm}$. Charged particles with a polar angle in the range $|\cos \theta|<0.88(0.95)$ traverse at least two (one) VDET layers. The VDET is surrounded by an inner tracking wire chamber (ITC) and by a large time projection chamber (TPC), which measures up to 21 three-dimensional coordinates per 
charged particle between radii of 31 and $180 \mathrm{~cm}$. The tracking achieves a momentum resolution $\sigma\left(p_{\mathrm{T}}\right) / p_{\mathrm{T}}$ of $6 \times 10^{-4} p_{\mathrm{T}} \oplus 0.005$, with $p_{\mathrm{T}}$ in $\mathrm{GeV} / c$. The resolution on the three-dimensional impact parameter of tracks can be parametrized as $(34+70 / p)\left(1+1.6 \cos ^{4} \theta\right) \mu \mathrm{m}$, with $p$ in $\mathrm{GeV} / c$.

The electromagnetic calorimeter (ECAL) is also situated inside the coil. It is segmented into projective towers of typically $0.9^{\circ} \times 0.9^{\circ}$, which allows electrons and photons to be identified within jets. Luminosity calorimeters of similar construction to ECAL are installed between the endcaps and the beam pipe and are treated as an extension of the ECAL. A silicon-tungsten sampling calorimeter extends the electromagnetic calorimeter coverage down to $34 \mathrm{mrad}$.

Outside the coil, a hadron calorimeter (HCAL) measures the hadronic energy, acts as a filter for the identification of muons, and serves as a return yoke for the magnetic field. The outermost detectors are two double layers of muon chambers.

The measurements of charged particle tracks and of energy deposits in the calorimeters, combined with the identification of photons, electrons, and muons, are used to produce a list of charged and neutral energy flow particles. Hadron jets, formed by clustering these particles, have an energy resolution of $\sigma(E)=(0.60 \sqrt{E}+0.6)\left(1+\cos ^{2} \theta\right)$, where $E$ is the energy in $\mathrm{GeV}$ and $\theta$ the polar angle of the jet. The resolution on the jet angles is approximately $20 \mathrm{mrad}$ in both $\theta$ and $\phi$.

The tagging of $\mathrm{b}$ quark jets is accomplished by combining several discriminating variables together using neural networks. These variables include an impact-parameter-based probability [8], a displaced vertex $\chi^{2}$ [9], and the transverse momentum of identified leptons with respect to the jet axis [10]. Three jet-shape quantities supplement these variables. The resulting six variable neural network b-tag is described in Ref. [11]; a more specialized four variable b-tag used for the $\mathrm{e}^{+} \mathrm{e}^{-} \rightarrow \mathrm{hA} \rightarrow \mathrm{b} \overline{\mathrm{b}} \mathrm{b} \overline{\mathrm{b}}$ final state is described in Ref. [1].

\section{$3 \quad$ Update of the searches}

\subsection{Signal and background simulation}

For each of the four centre-of-mass energies, fully simulated samples of signal and background events were generated. The HzHA program [12], used to generate the signal events, was also used to compute the signal cross sections, the Higgs boson decay branching fractions, and the radiative corrections to the Higgs boson masses in the MSSM. The most recent version contains, in particular, the latest refinements on these radiative corrections [3, 13, 14] and a more complete simulation of the he ${ }^{+} \mathrm{e}^{-}$and $\mathrm{h} \nu_{\mathrm{e}} \bar{\nu}_{\mathrm{e}}$ final states which now includes the interference between the boson fusion and the Higgsstrahlung processes [15].

The $\mathrm{e}^{+} \mathrm{e}^{-} \rightarrow \mathrm{q} \overline{\mathrm{q}}(\gamma)$ background was generated with KORALZ [16], instead of PYTHIA [17], to benefit from a more accurate treatment of multiple initial state photon radiation. The $\mathrm{e}^{+} \mathrm{e}^{-} \rightarrow \mathrm{We} \nu$ events, generated with PYTHIA, were compared to events generated with GRACE4F [18], and the differences were taken into account in the systematic uncertainties. The other Standard Model four-fermion processes were simulated in a manner similar to that described in Refs. [1, 2]. The sizes of the simulated signal and background samples correspond to at least 50 times the collected luminosity used in the analyses. 


\subsection{Event selections}

Analyses had already been developed [1, 2] for most final states relevant for the searches for neutral Higgs bosons. For the visible $\mathrm{e}^{+} \mathrm{e}^{-} \rightarrow \mathrm{hZ}$ search, these final states are the leptonic final state $\left(\mathrm{h} \ell^{+} \ell^{-}\right.$where $\ell$ denotes an electron or a muon), the missing energy final state $(\mathrm{h} \nu \bar{\nu})$, the four-jet final state (hq $\bar{q})$, and the tau final states $\left(h \tau^{+} \tau^{-}\right.$and $\left.h \rightarrow \tau^{+} \tau^{-}, \mathrm{Z} \rightarrow \mathrm{q} \overline{\mathrm{q}}\right)$. For the $\mathrm{e}^{+} \mathrm{e}^{-} \rightarrow$ hA search, they are the four-b final state $(\mathrm{b} \bar{b} \mathrm{~b} \bar{b})$ and the tau final state $\left(\mathrm{b} \bar{b} \tau^{+} \tau^{-}\right)$. For the invisible $\mathrm{e}^{+} \mathrm{e}^{-} \rightarrow \mathrm{hZ}$ search, they are the acoplanar lepton pair final state $\left(\mathrm{h} \ell^{+} \ell^{-}\right)$and the acoplanar jet pair final state (hq $\bar{q})$. As in Ref. [1], the visible Higgs boson search was conducted in two alternative analysis streams, a neural-network-based stream (NN) and a cut-based stream (cut), although both streams share the same leptonic and tau-final-state selections. This section highlights changes with respect to Refs. [1, 2]. Most of the hZ and hA selections were optimized for Higgs boson masses of 107 and $90 \mathrm{GeV} / c^{2}$, respectively, i.e., close to the search sensitivity in both cases.

A reoptimization of the selection criteria and retraining of the neural networks in all channels were performed to account for the higher centre-of-mass energies and integrated luminosity. In some cases, several new discriminating variables were adopted and refinements to analysis techniques implemented, as described below.

- In the missing energy channel, the reconstructed Higgs boson mass was removed from the variables of the single neural network selection (referred to as "A") in order to increase the selection efficiency for low Higgs boson masses. The structure of the three-neural-network selection (referred to as "B") remains unchanged.

- In the tau final state selection, the overlap with leptonic-final-state events $\left(\mathrm{he}^{+} \mathrm{e}^{-}\right.$or $\mathrm{h} \mu^{+} \mu^{-}$) was reduced by requiring that the measured invariant mass of the two tau jets be smaller than $75 \mathrm{GeV} / c^{2}$ when a decay particle of at least one of the two taus is identified as an electron or a muon.

- In the four-jet channels, the following improvements were made.

i) A four-constraint fit, identical to that used in Ref. [19], was adopted in all selections, instead of a simple energy rescaling. It allows the jet angles to be fitted as well as the jet energies, thus improving the dijet mass resolution, particularly near the kinematic threshold.

ii) In the four-b channel, a new variable was introduced to characterize the unbalanced topology of three jets recoiling against one. For each of the possible three-jet combinations, the energy-weighted angular dispersion is computed as

$$
\Delta_{i j k} \equiv\left(\sum_{l \in \text { jet } i, j, k} \theta_{l, \vec{u}}^{2} E_{l} / \sum_{l \in \text { jet } i, j, k} E_{l}\right)^{1 / 2}
$$

where the indices $i, j$, and $k$ refer to jets, $\theta_{l, \vec{u}}$ is the angle between the momentum direction of the object $l$ contained in jet $i, j, k$ and the vectorial sum $\vec{u}$ of the three jet momenta, and $E_{l}$ is the energy of object $l$. The variable $\Delta \theta_{3}$ is defined as the minimum value of this dispersion for all four possible three-jet systems. 


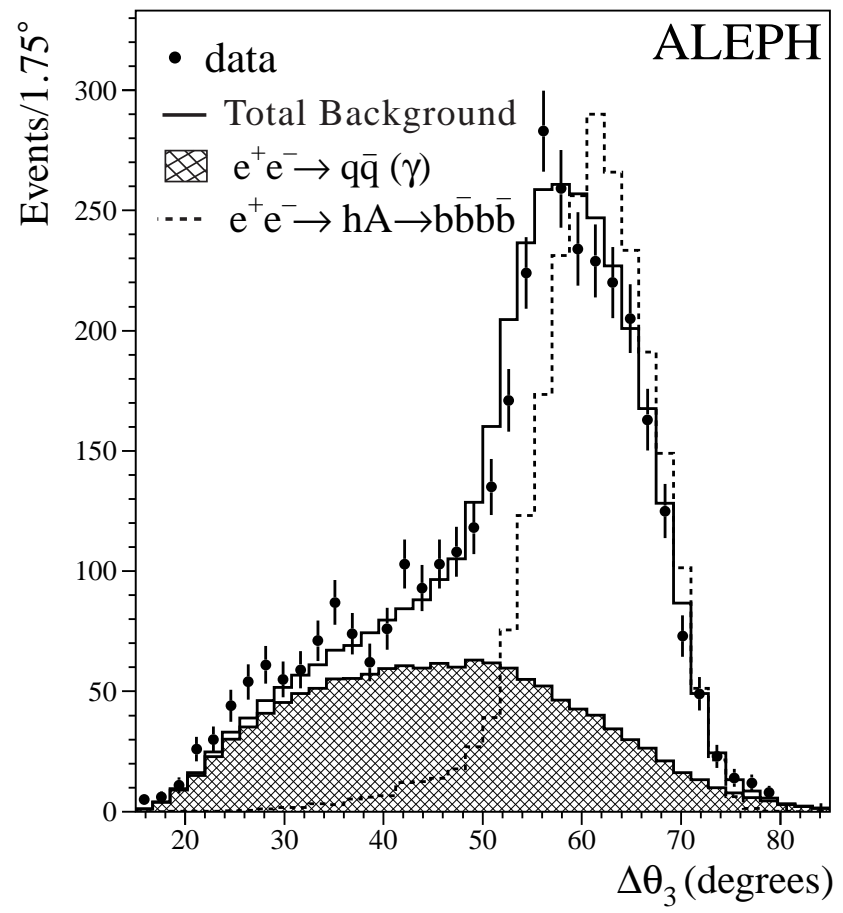

Figure 1: Distribution, at preselection level, of the minimum value of the energy-weighted angular dispersion $\Delta \theta_{3}$ in the four-b channel for data (dots with error bars), simulated background (solid histogram), and the signal for $m_{\mathrm{h}}=m_{\mathrm{A}}=90 \mathrm{GeV} / c^{2}$ (dashed histogram). The signal histogram has an arbitrary normalization.

The distribution of this quantity is shown in Fig. 1. The cut $\Delta \theta_{3}>50^{\circ}$ reduces the background from events with gluon splitting $\mathrm{e}^{+} \mathrm{e}^{-} \rightarrow \mathrm{b} \overline{\mathrm{b}}(\mathrm{g} \rightarrow \mathrm{b} \overline{\mathrm{b}})$.

iii) In the neural network selection, the former 17 variables were supplemented with the decay angles relative to the direction of flight of the $\mathrm{Z}$ and Higgs boson candidates, $\alpha_{12}$ and $\alpha_{34}$. The output of the neural network for the four-jet final state is shown in Fig. 2 .

\subsection{Systematic uncertainties}

Systematic uncertainties caused by inaccuracies of the simulation were evaluated as in Refs. [1, 2]. Whenever possible, they were extracted from $3.5 \mathrm{pb}^{-1}$ of data taken at the $\mathrm{Z}$ peak during the same year. A discrepancy between data and simulation, affecting the acceptance for signal and for most of the background processes, was observed in the impact-parameterbased b tagging quantities. To correct for this effect, a smearing of the track parameters was performed on the simulated events to bring them in better agreement with the data. Half of the correction was conservatively taken as a systematic uncertainty for all channels. The systematic uncertainties on signal efficiencies are typically $5 \%$, and those on the background yields are summarized in Table 1 .

\section{Combination and results}




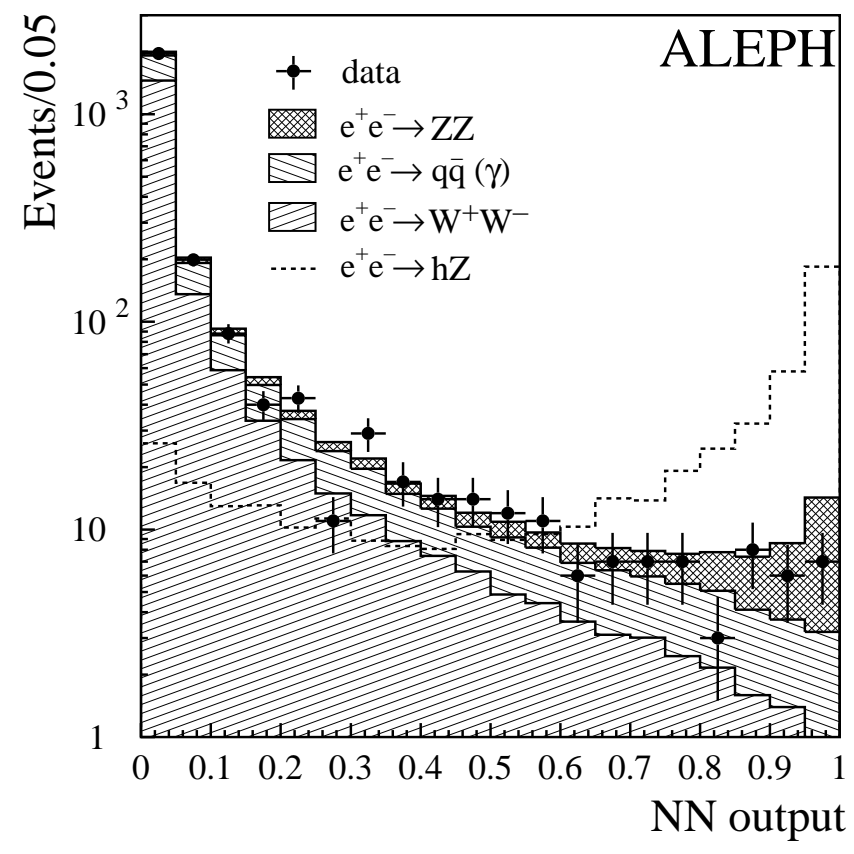

Figure 2: Distributions of the neural network output used to select four-jet hZ candidates for data (dots with error bars), simulated background (solid histograms), and simulated Higgs signal with an arbitrary normalization for $m_{\mathrm{h}}=107 \mathrm{GeV} / c^{2}$ (dashed histogram), after preselection criteria have been applied.

\subsection{Selection overlaps}

Events selected by more than one analysis were treated with a well-defined prescription to remove overlaps. This procedure prevents any given event from affecting the confidence level calculations more than once. The potential overlaps of the searches for different topologies arising from the Higgsstrahlung process were suppressed by assigning a precedence order to the four selections, and by rejecting any candidate event already selected by a higher precedence search. The order of the precedence was chosen to be 1) the missing energy final state; 2) the tau final states; 3) the leptonic final state; and 4) the four-jet final state. In the data, no event was affected by this procedure.

The significant overlaps of the $\mathrm{hZ}$ and hA searches for tau final states as well as for four-jet final states in the cut stream were treated in a manner similar to that used in Ref. [1] for the missing energy channel. This method divides the analyses into three statistically independent branches: one branch containing the events selected only by the hZ search; a second branch containing the events selected only by the hA search; and a third branch containing the events selected simultaneously by both searches. The use of different reconstructed Higgs boson mass definitions in the $\mathrm{hZ}$ searches (with the $\mathrm{Z}$ mass constraint) and the hA searches (with an equal mass constraint) introduces an additional difficulty, solved in the following manner. The hZ (hA) mass definition is used for the hZ(hA)-only branch. For the third branch, the mass definition depends on the MSSM parameter set and is chosen such that it maximizes the combined expected 95\% CL sensitivity. Typically, the hZ mass definition is chosen for small $\tan \beta$ values, where the hZ cross section dominates, and the hA mass definition is chosen for large $\tan \beta$ values. 
Table 1: The numbers of signal and background events expected, and the numbers of candidate events observed in the data for each channel. The signal expectation is computed for the hZ process with a Higgs boson mass of $107 \mathrm{GeV} / c^{2}$, and that for the hA process with a common Higgs boson mass of $90 \mathrm{GeV} / c^{2}$. The background yield is subdivided into $\mathrm{ZZ}$ (including Zee and $\mathrm{Z} \nu \bar{\nu}$ ), WW (including We $\nu$ ), and $\mathrm{f} \overline{\mathrm{f}}$ (including $\gamma \gamma \rightarrow \mathrm{f} \overline{\mathrm{f}}$ ). The f $\overline{\mathrm{f}}$ composition is entirely $\mathrm{q} \overline{\mathrm{q}}$ for the visible Higgs boson and hadronic invisible Higgs boson decay channels, and $\ell^{+} \ell^{-}$and $\gamma \gamma \rightarrow \ell^{+} \ell^{-}$for the leptonic invisible Higgs boson decay channel. Systematic uncertainties are given for each background.

\begin{tabular}{|c|c|c|c|c|c|c|c|c|}
\hline \multirow{2}{*}{\multicolumn{2}{|c|}{ Analyses }} & \multicolumn{2}{|c|}{$\begin{array}{c}\text { Signal } \\
\text { expected }\end{array}$} & \multicolumn{4}{|c|}{$\begin{array}{c}\text { Background } \\
\text { expected }\end{array}$} & \multirow[t]{2}{*}{$\begin{array}{c}\text { Events } \\
\text { Observed }\end{array}$} \\
\hline & & $\mathrm{hZ}$ & $\mathrm{hA}$ & $\mathrm{ZZ}$ & WW & $\overline{f f}$ & Total & \\
\hline \multicolumn{2}{|c|}{$\mathrm{h} \ell^{+} \ell^{-}$} & 0.8 & - & $25.4 \pm 0.3$ & $1.9 \pm 0.1$ & $1.2 \pm 0.2$ & $28.5 \pm 0.4$ & 26 \\
\hline \multicolumn{2}{|c|}{$\mathrm{h} \nu \bar{\nu}$ (Cut) } & 2.6 & $\begin{array}{l}- \\
-\end{array}$ & $11.1 \pm 1.1$ & $10.3 \pm 3.1$ & $3.7 \pm 2.2$ & $25.1 \pm 4.0$ & 23 \\
\hline \multirow{3}{*}{$\begin{array}{l}\mathrm{h} \nu \bar{\nu} \\
(\mathrm{NN})\end{array}$} & $\mathrm{A} \& \mathrm{~B}$ & 1.1 & - & $2.5 \pm 0.1$ & $0.2 \pm 0.0$ & $0.5 \pm 0.1$ & $3.2 \pm 0.1$ & 3 \\
\hline & A only & 0.6 & - & $4.2 \pm 0.1$ & $1.0 \pm 0.1$ & $0.6 \pm 0.1$ & $5.8 \pm 0.2$ & 3 \\
\hline & B only & 0.2 & - & $0.5 \pm 0.0$ & $0.1 \pm 0.0$ & $1.4 \pm 0.2$ & $2.0 \pm 0.2$ & 1 \\
\hline \multirow{3}{*}{$\begin{array}{l}\text { hq̄ } \\
\text { (Cut) }\end{array}$} & $\overline{\mathrm{hZ} \& \mathrm{hA}}$ & 1.5 & 2.4 & $\overline{5.0 \pm 0.4}$ & $0.3 \pm 0.1$ & $2.5 \pm 0.4$ & $\overline{7.8 \pm 0.8}$ & 3 \\
\hline & hZ only & 2.2 & 0.3 & $10.8 \pm 0.6$ & $2.9 \pm 0.3$ & $5.9 \pm 0.5$ & $19.7 \pm 1.0$ & 16 \\
\hline & hA only & 0.3 & 1.2 & $2.3 \pm 0.2$ & $1.2 \pm 0.2$ & $5.3 \pm 0.6$ & $8.8 \pm 0.8$ & 8 \\
\hline \multirow{2}{*}{$\begin{array}{c}\text { hqव } \\
(\mathrm{NN})\end{array}$} & $2 \mathrm{~b}$ & 4.1 & 1.0 & $19.7 \pm 1.0$ & $7.7 \pm 0.6$ & $12.3 \pm 1.5$ & $239.7 \pm 1.9$ & 27 \\
\hline & $4 \mathrm{~b}$ & 1.1 & 2.2 & $3.9 \pm 0.3$ & $0.3 \pm 0.1$ & $2.5 \pm 0.7$ & $6.7 \pm 0.8$ & 3 \\
\hline \multirow{3}{*}{$\begin{array}{c}\mathrm{b} \overline{\mathrm{b}} \tau^{+} \tau^{-} \\
\& \\
\tau^{+} \tau^{-} \mathrm{q} \overline{\mathrm{q}}\end{array}$} & hZ \& hA & 0.2 & 0.4 & $1.8 \pm 0.2$ & $0.4 \pm 0.1$ & $0.1 \pm 0.1$ & $2.3 \pm 0.2$ & 0 \\
\hline & hZ only & 0.3 & 0.1 & $4.3 \pm 0.2$ & $4.6 \pm 0.2$ & $0.8 \pm 0.2$ & $9.7 \pm 0.3$ & 13 \\
\hline & hA only & 0.0 & 0.0 & $0.2 \pm 0.0$ & $0.1 \pm 0.0$ & $0.1 \pm 0.1$ & $0.4 \pm 0.1$ & 2 \\
\hline \multicolumn{2}{|c|}{ invisible $\mathrm{h} \ell^{+} \ell^{-}$} & 0.3 & - & $3.4 \pm 0.4$ & $3.7 \pm 0.5$ & $1.4 \pm 0.2$ & $8.5 \pm 0.7$ & 8 \\
\hline \multicolumn{2}{|c|}{ invisible $h q \bar{q}$} & 3.3 & $\begin{array}{c}- \\
-\end{array}$ & $31.8 \pm 0.8$ & $17.4 \pm 3.5$ & $3.1 \pm 1.2$ & $252.3 \pm 3.8$ & $\overline{50}$ \\
\hline
\end{tabular}

The three-branch subdivision in the NN stream four-jet final state was replaced by a simpler and similarly sensitive two-branch treatment: the first branch (4b) containing the events selected by the four-b final state search, and the second branch (2b) containing the events not selected by the $4 \mathrm{~b}$ search.

\subsection{Selection results}

For each analysis, the numbers of signal and background events expected, and the observed number of events are summarized in Table 1. For the Standard Model Higgs boson searches, a total of 76 events are selected in the data with the NN stream, while 97.8 events are expected from Standard Model processes. Figure 3 shows the reconstructed Higgs boson mass distributions for the data and the background expectations for the hZ selections. The hA selections yield 5 events in the data with the NN stream, while 9.4 events are expected from Standard Model processes. In the search for invisibly decaying Higgs bosons, a total of 58 candidate events are selected with 60.8 events expected from Standard Model processes; the distributions of their reconstructed Higgs boson mass are shown in Fig. $⿴$. 

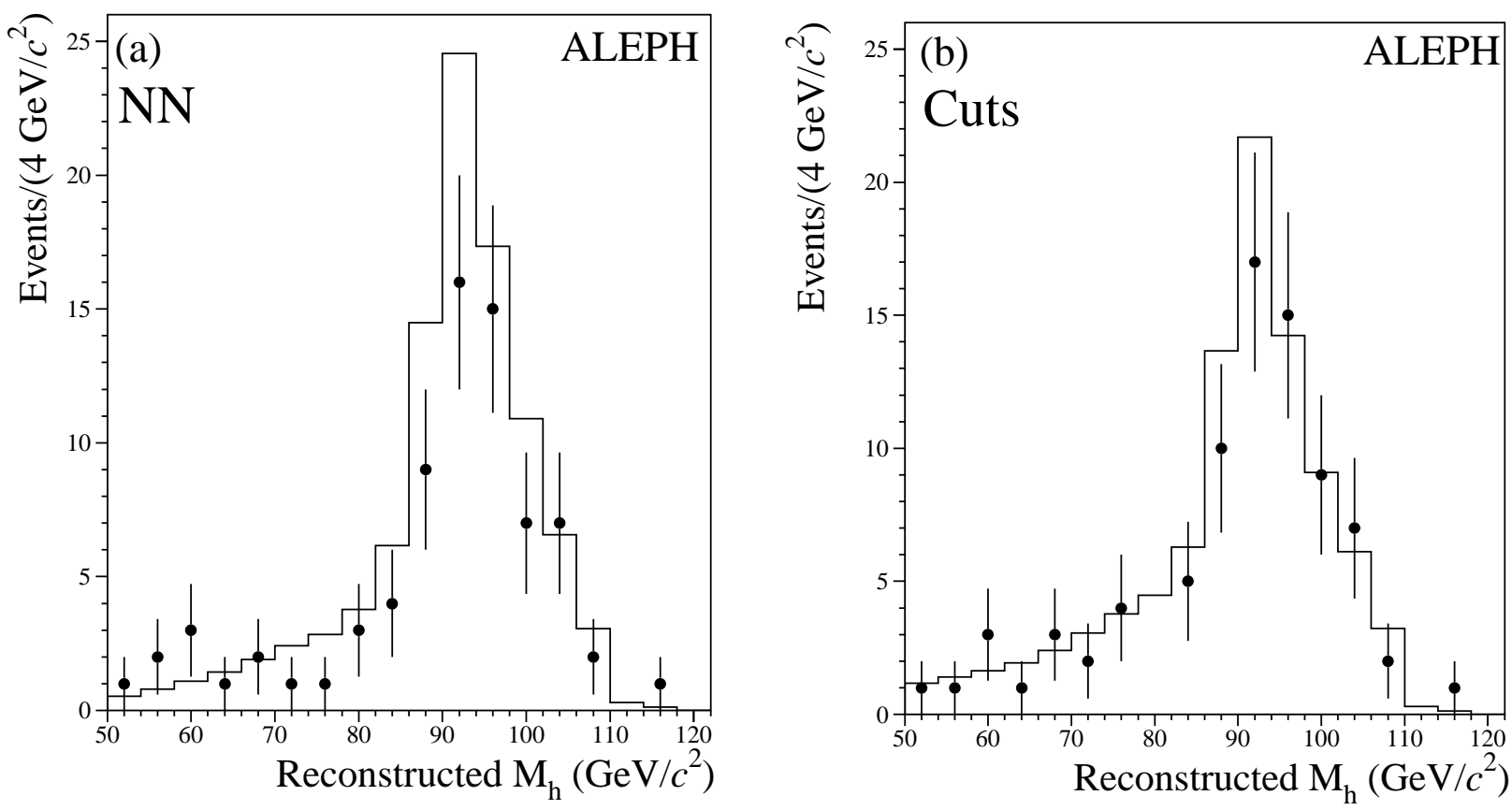

Figure 3: Distributions of the reconstructed Higgs boson mass $M_{\mathrm{h}}$ for the data (dots with error bars) selected in the hZ searches by (a) the NN stream, and (b) the cut stream. The histograms show the Standard Model background expectation.

Fewer events were selected in the data than were expected. This deficit is mostly due to the four-jet analysis of the NN stream where 46.4 events were expected, but only 30 events were selected in the data. In the four-jet cut analysis 27 events are selected in data with 36.3 expected. As can be seen in Fig. 3, the deficit of events is mostly near the mass region dominated by $\mathrm{e}^{+} \mathrm{e}^{-} \rightarrow \mathrm{ZZ}$ events.

At the preselection level, the background expectation is dominated by four-jet $\mathrm{e}^{+} \mathrm{e}^{-} \rightarrow \mathrm{W}^{+} \mathrm{W}^{-}$events which are kinematically similar to signal events, and no significant discrepancies were found between data and Monte Carlo simulation for all relevant kinematic variables. Figure 2 shows the distribution of the neural network output distribution at the preselection level. Overall, the agreement between the simulated expectation and the data is very good with 2572 events expected and 2523 seen in the data. The deficit is, however, apparent in Fig. 2 at high values of the neural network output, which is dominated by b tagged $\mathrm{e}^{+} \mathrm{e}^{-} \rightarrow$ ZZ events.

As mentioned in Section 3.3, a slight disagreement was found and corrected for in the $\mathrm{b}$ tagging distributions. The corrections were determined from $\mathrm{Z}$ peak data taken during the same year. These were verified with $\mathrm{e}^{+} \mathrm{e}^{-} \rightarrow \mathrm{q} \overline{\mathrm{q}} \gamma$ radiative return events and semileptonic $\mathrm{e}^{+} \mathrm{e}^{-} \rightarrow \mathrm{W}^{+} \mathrm{W}^{-} \rightarrow \mathrm{q} \overline{\mathrm{q}} \ell \nu$ events collected at high centre-of-mass energies. The $\mathrm{q} \overline{\mathrm{q}} \gamma$ events provide a known $b \bar{b}$ content while the semileptonic $\mathrm{W}^{+} \mathrm{W}^{-}$events provide a control sample with negligible b-jet content. The impact of these b-tagging corrections is propagated to the simulation, but the effect is too small to account for the observed deficit of events. The conclusion of these studies is that the cause of the deficit is most likely due to a statistical fluctuation. 


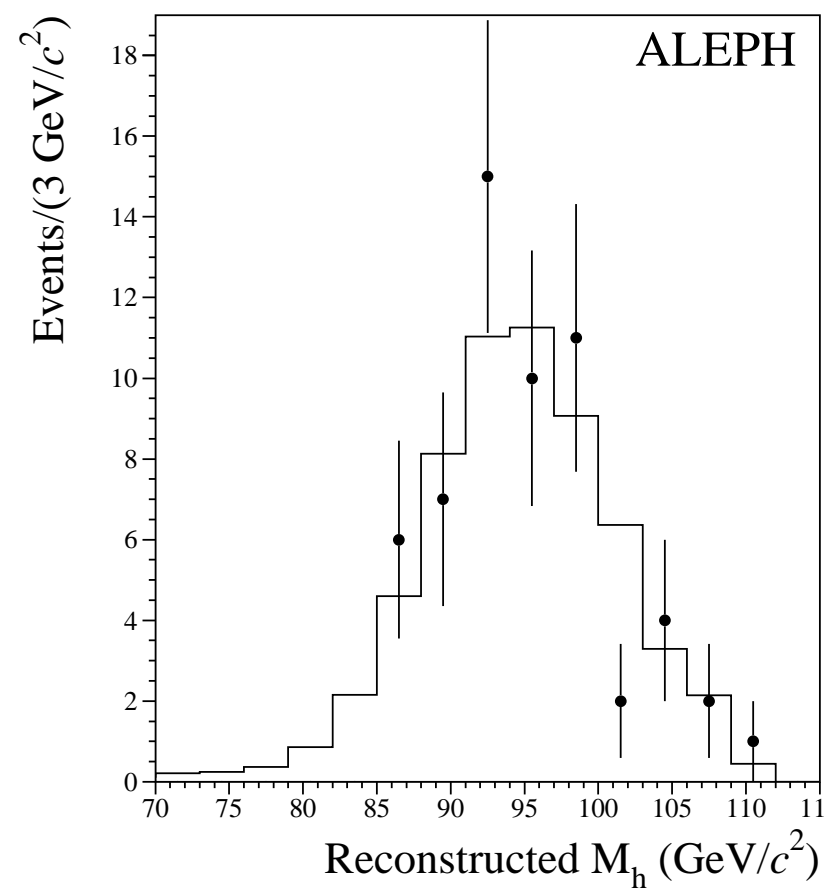

Figure 4: Distribution of the reconstructed Higgs boson mass $M_{\mathrm{h}}$ for the data (dots with error bars) and the expected background (histogram) in the invisibly decaying Higgs boson searches for the acoplanar lepton and the acoplanar jet final states.

\subsection{Exclusion limits}

The $176.2 \mathrm{pb}^{-1}$ of data collected in 1998 with a centre-of-mass energy of $188.6 \mathrm{GeV}$ were combined with the 1999 data to determine the final results. As was done in Refs. [1], 2], confidence levels on the signal hypothesis were drawn using the Signal Estimator method [20] rather than with the method used by the other LEP experiments [21], thus increasing the expected mass limit by $\sim 0.5 \mathrm{GeV} / c^{2}$. Expected confidence levels were calculated as the median confidence level in the absence of signal.

The tau final state analyses and the cut analyses for the four-jet, four-b, and missing energy topologies use only the reconstructed Higgs boson mass as a discriminant in the confidence level calculations. The other analyses use an additional discriminant variable: the neural network output in the case of the $2 \mathrm{~b}$ branch of the $\mathrm{NN}$ stream four-jet analysis and a b-tag sensitive variable in the $4 \mathrm{~b}$ branch as well as in the $\mathrm{h} \ell^{+} \ell^{-}$final state analysis.

The expected and observed confidence levels are shown in Fig. 0 for the Standard Model Higgs boson searches. The low confidence levels for the background hypothesis at small Higgs boson masses are a reflection of the observed deficit of events discussed in Section 1.2. The lower limits at $95 \%$ confidence level on the mass of the Standard Model Higgs boson with systematic uncertainties taken into account [22] are summarized in Table 2. Since the NN stream gives an expected Higgs boson mass lower limit higher by about $0.5 \mathrm{GeV} / c^{2}$, it is used to set the final results.

Figure 6 shows the hZ, the $\mathrm{hA}$, and their combined Higgs boson mass exclusions as a function 
Table 2: Summary of the 95\% CL lower limits on Higgs boson masses in the Standard Model and in the MSSM, and their expected values in absence of signal. The $\tan \beta$ excluded ranges in the $m_{\mathrm{h}}^{\max }$ scenario are also given for a top quark mass of $175 \mathrm{GeV} / c^{2}$.

\begin{tabular}{|c||c|c||c|c|}
\hline \hline \multicolumn{1}{|c||}{} & \multicolumn{3}{c|}{$95 \%$ CL lower limits $\left(\mathrm{GeV} / c^{2}\right)$} \\
\cline { 2 - 5 } & \multicolumn{2}{c|}{ NN stream } & \multicolumn{2}{c|}{ Cut stream } \\
\cline { 2 - 5 } & Median Expected & Observed & Median Expected & Observed \\
\hline SM $m_{\mathrm{h}}$ & 107.8 & 107.7 & 107.3 & 105.2 \\
\hline MSSM $m_{\mathrm{h}}$ & 88.7 & 91.2 & 88.3 & 90.7 \\
MSSM $m_{\mathrm{A}}$ & 89.1 & 91.6 & 88.7 & 91.1 \\
\hline $\tan \beta$ & {$[0.8,1.9]$} & {$[0.8,1.9]$} & {$[0.8,1.9]$} & {$[0.8,1.7]$} \\
\hline \hline
\end{tabular}

of $\sin ^{2}(\beta-\alpha)$. These combined exclusions are interpreted within two benchmark parameter sets, one which maximizes the radiative corrections to the lighter CP-even Higgs boson mass as a function of $\tan \beta$ (referred to as the $m_{\mathrm{h}}^{\max }$ scenario) 14 and one in which no mixing in the scalar top sector is assumed. The excluded domains in the $\left[m_{\mathrm{h}}, \tan \beta\right]$ plane are shown in Fig. 7 as obtained using the NN stream. The results in the $m_{\mathrm{h}}^{\max }$ scenario are summarized in Table 2 . The $\tan \beta$ exclusion range is sensitive to the top quark mass assumption which was taken to be $175 \mathrm{GeV} / c^{2}$. For a top quark mass of $180 \mathrm{GeV} / c^{2}$, the $\tan \beta$ exclusion range reduces to $[1.0,1.5]$.

For the invisibly decaying Higgs boson search, no discriminant variable is used in the confidence level calculations, as was done in the previous search at $188.6 \mathrm{GeV}$ [2], because the sliding analysis technique effectively acts as if the reconstructed mass were used as a discriminant variable. The results are interpreted as an exclusion domain in the $\left(m_{\mathrm{h}}, \xi^{2}\right)$ plane, presented in Fig. 8. For $\xi^{2}=1$, invisibly decaying Higgs bosons with masses below $106.4 \mathrm{GeV} / c^{2}$ are excluded at $95 \% \mathrm{CL}$ with an expected limit of $105.6 \mathrm{GeV} / c^{2}$.

\section{Conclusions}

Searches for neutral Higgs bosons in $\mathrm{e}^{+} \mathrm{e}^{-}$collisions at centre-of-mass energies from 191.6 to 201.6 GeV have been carried out with the ALEPH detector using an integrated luminosity of $237 \mathrm{pb}^{-1}$. The major event topologies expected from the hZ process for visibly as well as invisibly decaying Higgs bosons, the weak boson fusion process, and the hA process have been analysed. The data were combined with the $176 \mathrm{pb}^{-1}$ collected in 1998 at a centre-of-mass energy of $188.6 \mathrm{GeV}$.

A $95 \%$ CL lower limit on the Standard Model Higgs boson mass of $107.7 \mathrm{GeV} / c^{2}$ is obtained with an expected sensitivity of $107.8 \mathrm{GeV} / c^{2}$. Lower limits of 91.2 and $91.6 \mathrm{GeV} / c^{2}$ are obtained at 95\% CL for the masses of the $\mathrm{h}$ and A neutral Higgs bosons of the MSSM. These limits, determined in the $m_{\mathrm{h}}^{\max }$ scenario, are valid for any $\tan \beta>0.5$. For a top quark mass of $175 \mathrm{GeV} / c^{2}$, the $\tan \beta$ range between 0.8 and 1.9 is excluded with $95 \%$ confidence.

An invisibly decaying Higgs boson with a production cross section equal to that in the Standard Model is excluded at 95\% CL for masses below $106.4 \mathrm{GeV} / c^{2}$. 

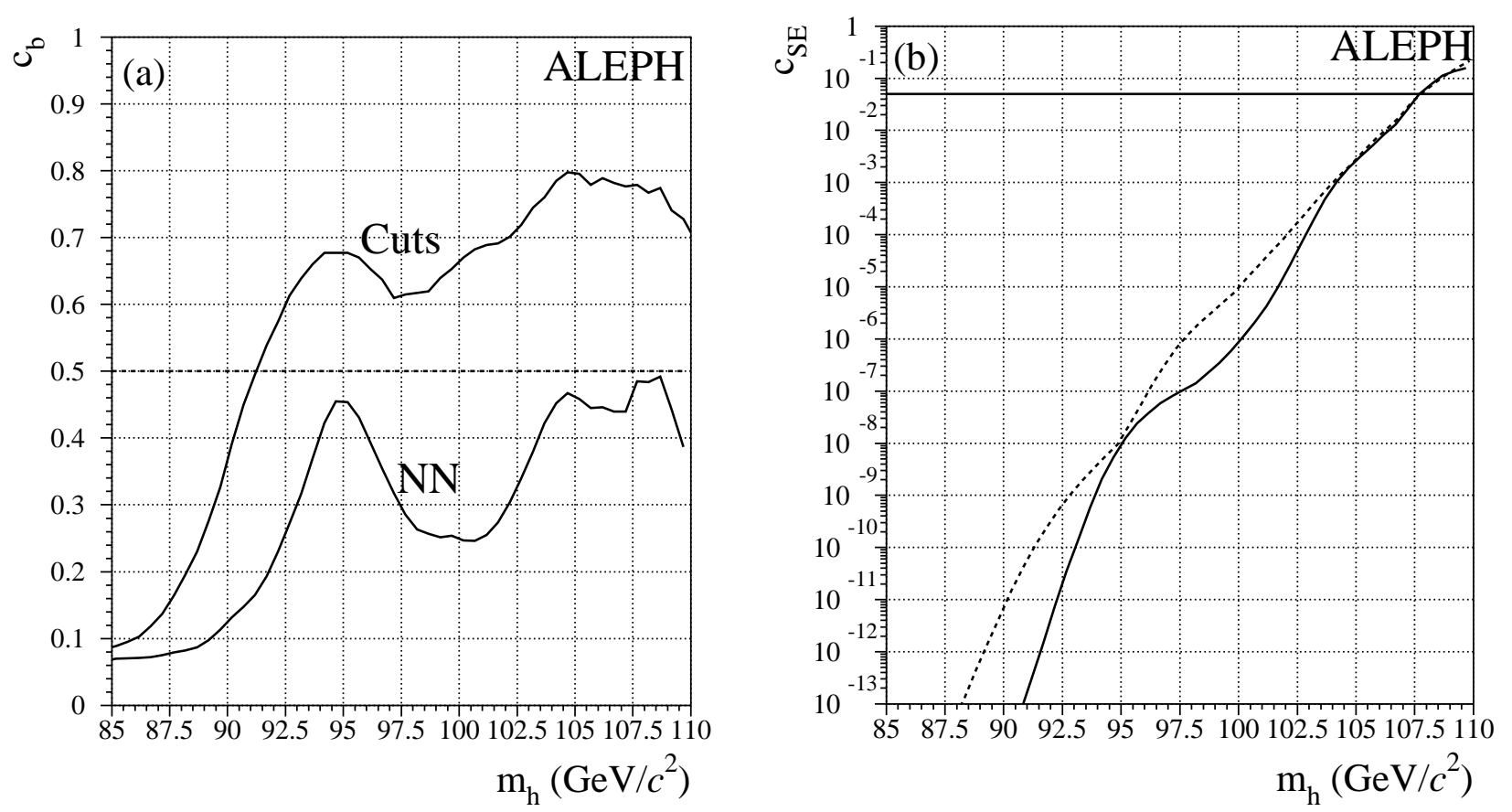

Figure 5: Observed (solid) and expected (dashed) CL curves for (a) the background hypothesis and (b) the signal hypothesis for the NN stream, as functions of the hypothesized Standard Model Higgs boson mass $m_{\mathrm{h}}$. For the signal hypothesis (b), the intersections of the horizontal line at $5 \%$ with the curves define the observed and expected 95\% CL lower limits on the Standard Model Higgs boson mass.
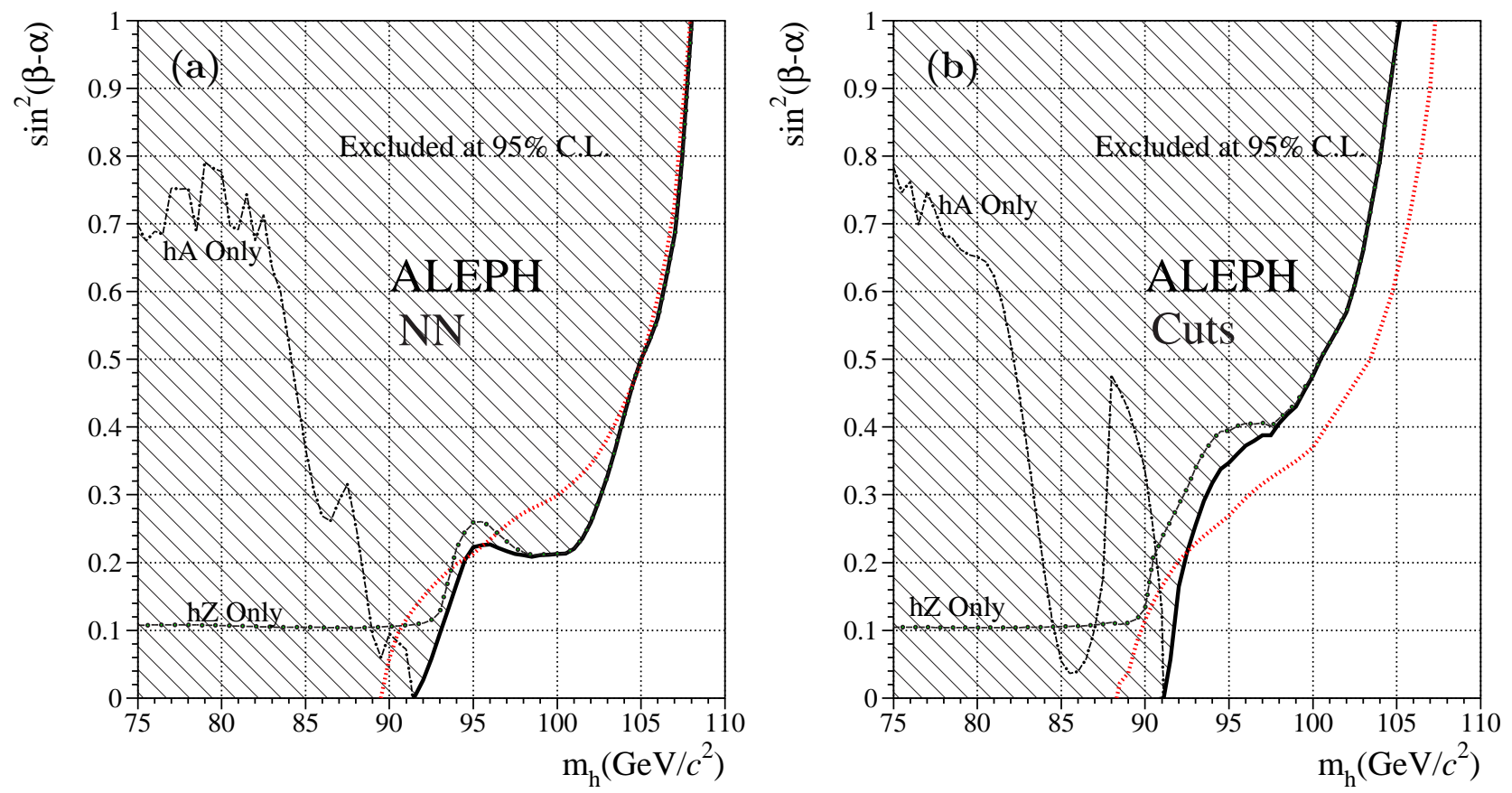

Figure 6: The expected (dashed) and observed (solid) excluded regions at 95\% CL in the plane $\left[m_{\mathrm{h}}, \sin ^{2}(\beta-\alpha)\right]$ for $(\mathrm{a})$ the NN stream and (b) the cut stream. 

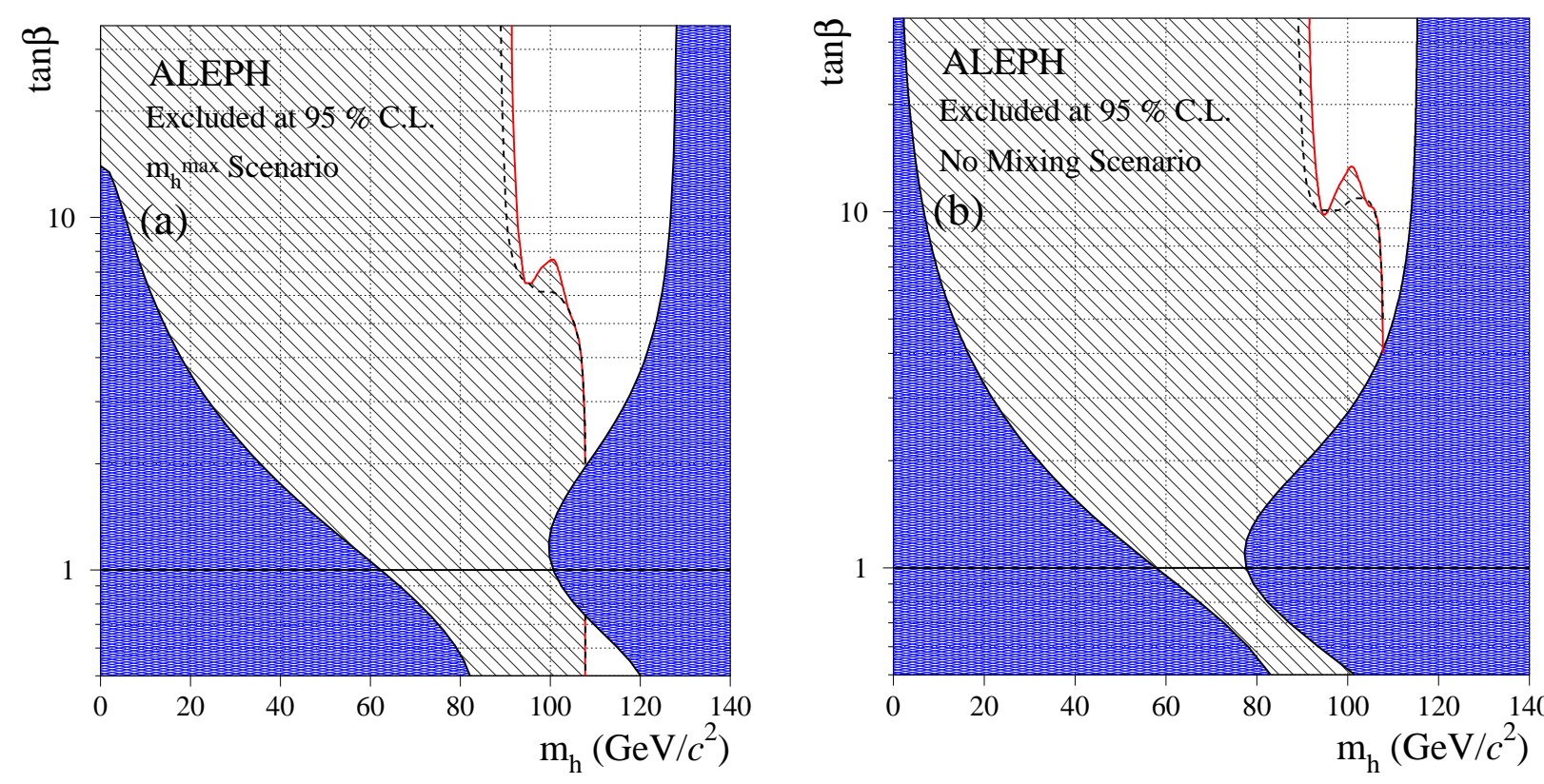

Figure 7: The expected (dashed) and observed (solid) excluded regions at 95\% CL for the NN stream in the $\left[m_{\mathrm{h}}, \tan \beta\right]$ plane of the MSSM for (a) the $m_{\mathrm{h}}^{\max }$ parameter set and (b) the no mixing scenario. The densely hatched regions are not allowed theoretically.

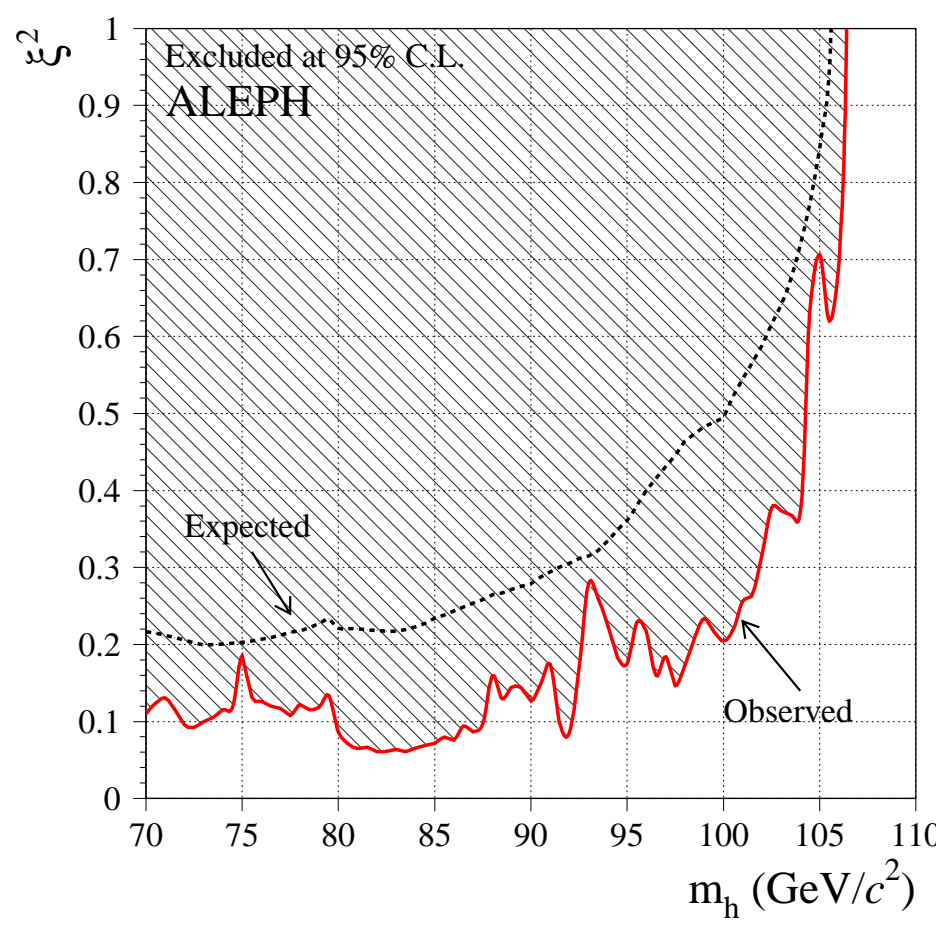

Figure 8: Observed (solid) and expected (dashed) regions excluded at the 95\% CL in the $\left(m_{\mathrm{h}}, \xi^{2}\right)$ plane for an invisibly decaying Higgs boson. 


\section{Acknowledgements}

We wish to congratulate our colleagues from the accelerator divisions for the very successful operation of LEP at high energies. We are indebted to the engineers and technicians in all our institutions for their contribution to the excellent performance of ALEPH. Those of us from non-member countries thank CERN for its hospitality.

\section{References}

[1] ALEPH Collaboration, Search for the neutral Higgs bosons of the Standard Model and the MSSM in $e^{+} e^{-}$collisions at $\sqrt{s}=189$ GeV, CERN-EP/2000-019 (2000), submitted to Eur. Phys. J. C.

[2] ALEPH Collaboration, Search for an invisibly decaying Higgs boson in $e^{+} e^{-}$collisions at 189 GeV, Phys. Lett. B466 (1999) 50.

[3] M. Carena, P.M. Zerwas et al., Higgs physics, in "Physics at LEP2", Eds. G. Altarelli, T. Sjöstrand, and F. Zwirner, CERN 96-01 (1996), p. 351.

[4] DELPHI Collaboration, A search for invisible Higgs bosons produced in $e^{+} e^{-}$interactions up to $\sqrt{s}=189$ GeV, CERN-EP/2000-051 (2000), submitted to Phys. Lett. B;

DELPHI Collaboration, Searches for neutral Higgs bosons in $e^{+} e^{-}$collisions around $\sqrt{s}=189$ GeV, CERN-EP/2000-038 (2000), submitted to Eur. Phys. J. C;

L3 Collaboration, Search for the Standard Model Higgs boson in $e^{+} e^{-}$interactions at $\sqrt{s}=189$ GeV, Phys. Lett. B461 (1999) 376;

L3 Collaboration, Search for neutral Higgs bosons of the Minimal Supersymmetric Standard Model in $e^{+} e^{-}$interactions at $\sqrt{s}=189 \mathrm{GeV}$, Phys. Lett. B471 (1999) 321;

L3 Collaboration, Search for an invisibly decaying Higgs boson in $e^{+} e^{-}$collisions at $\sqrt{s}=183-189$ GeV, Phys. Lett. B485 (2000) 85;

OPAL Collaboration, Search for neutral Higgs bosons in $e^{+} e^{-}$collisions at $\sqrt{s} \sim 189$ GeV, E. Phys. J. C12 (2000) 567.

[5] ALEPH Collaboration, ALEPH: A detector for electron-positron annihilations at LEP, Nucl. Instrum. and Methods A294 (1990) 121.

[6] ALEPH Collaboration, Performance of the ALEPH detector at LEP, Nucl. Instrum. and Methods A360 (1995) 481.

[7] D. Creanza et al., The new ALEPH vertex detector, Nucl. Instrum. and Methods A409 (1998) 157.

[8] ALEPH Collaboration, A precise measurement of $\Gamma_{Z \rightarrow b \bar{b}} / \Gamma_{Z \rightarrow \text { hadrons, }}$ Phys. Lett. B313 (1993) 535.

[9] ALEPH Collaboration, Limit on $B_{s}^{0}$ oscillation using a jet charge method, Phys. Lett. B356 (1995) 409; An investigation of $B_{d}^{0}$ and $B_{s}^{0}$ oscillation, Phys. Lett. B322 (1994) 441. 
[10] ALEPH Collaboration, Heavy quark tagging with leptons in the ALEPH detector, Nucl. Instrum. and Methods A436 (1994) 461.

[11] ALEPH Collaboration, Search for the neutral Higgs bosons of the MSSM in $e^{+} e^{-}$collisions at $\sqrt{s}$ from 130 to $172 \mathrm{GeV}$, Phys. Lett. B412 (1997) 173.

[12] P. Janot, The HZHA generator, in "Physics at LEP", Eds. G. Altarelli, T. Sjöstrand and F. Zwirner, CERN 96-01 (1996) Vol. 2, p. 309;

For a description of the updates and for the code, see: http://alephwww.cern.ch/ janot/Generators.html

[13] M. Carena, J.R. Espinosa, M. Quirós, and C.E.M. Wagner, Analytical expressions for radiatively corrected Higgs masses and couplings in the MSSM, Phys. Lett. B355 (1995) 209 ;

S. Heinemeyer, W. Hollik, and G. Weiglein, FeynHiggs: a program for the calculation of the masses of the neutral $\mathcal{C P}$-even Higgs bosons in the MSSM, Comput. Phys. Commun. 124 (2000) 76.

[14] M. Carena, S. Heinemeyer, C.E.M. Wagner, and G. Weiglein, Suggestions for improved benchmark scenarios for Higgs-boson searches at LEP 2, CERN-TH/99-374 (1999).

[15] F. A. Berends and R. Kleiss, Initial state radiation at LEP energies and the corrections to Higgs boson production, Nucl. Phys. B260 (1985) 32;

W. Kilian, M. Kramer and P.M. Zerwas, Higgstrahlung and WW fusion in $e^{+} e^{-}$collisions, Phys. Lett. B373 (1996) 135; Higgsstrahlung and vector boson fusion in $e^{+} e^{-}$collisions, hep-ph/9605437 (1996).

[16] S. Jadach, B.F.L. Ward, and Z. Wạs, The Monte Carlo program KORALZ version 4.0 for the lepton or quark pair production at LEP/SLC energies, Comput. Phys. Commun. 79 (1994) 503.

[17] T. Sjöstrand, High-energy-physics event generation with PYTHIA 5.7 and JETSET \%.4, Comput. Phys. Commun. 82 (1994) 74.

[18] J. Fujimoto et al., GRC4F V1.1: A four fermion event generator for $e^{+} e^{-}$collisions, Comput. Phys. Commun. 100 (1997) 128.

[19] ALEPH Collaboration, Measurement of the $W$ mass by direct reconstruction in $e^{+} e^{-}$ collisions at 172 GeV, Phys. Lett. B422 (1998) 384.

[20] S. Jin and P. McNamara, The signal estimator limit setting method, in "Workshop on Confidence Limits", Eds. F. James, L. Lyons, and Y. Perrin, CERN 2000-005 (2000) p. 103.

[21] A. L. Read, Modified frequentist analysis of search results (the $C L_{s}$ method) in "Workshop on Confidence Limits", Eds. F. James, L. Lyons, and Y. Perrin, CERN 2000-005 (2000) p. 81.

[22] R. D. Cousins and V. L. Highland, Incorporating systematic uncertainties into an upper limit, Nucl. Instrum. and Methods A320 (1992) 331. 